EPJ Web of Conferences 113, 08003 (2016)

DOI: $10.1051 /$ epjconf/201611308003

(C) Owned by the authors, published by EDP Sciences, 2016

\title{
Low-energy calculations for nuclear photodisintegration
}

\author{
S. Deflorian ${ }^{1,2, a}$, V.D. Efros ${ }^{3}$, and W. Leidemann ${ }^{1,2}$ \\ ${ }^{1}$ Dipartimento di Fisica, Università di Trento, I-38123 Trento, Italy \\ ${ }^{2}$ Istituto Nazionale di Fisica Nucleare, TIFPA, I-38123 Trento, Italy \\ ${ }^{3}$ Russian Research Centre "Kurchatov" Institute, Kurchatov Square, 123182 Moscow, Russia
}

\begin{abstract}
In the Standard Solar Model a central role in the nucleosynthesis is played by reactions of the kind ${ }_{Z_{1}}^{A_{1}} X_{1}+{ }_{Z_{2}}^{A_{2}} X_{2} \rightarrow{ }_{Z_{1}+Z_{2}}^{A_{1}+A_{2}} Y+\gamma$, which enter the proton-proton chains. These reactions can also be studied through the inverse photodisintegration reaction. One option is to use the Lorentz Integral Transform approach, which transforms the continuum problem into a bound state-like one. A way to check the reliability of such methods is a direct calculation, for example using the Kohn Variational Principle to obtain the scattering wave function and then directly calculate the response function of the reaction.
\end{abstract}

Our goal is to study nuclear reactions of the kind

$$
{ }_{Z}^{A} N+\gamma \rightarrow X_{1}+X_{2}
$$

close to the threshold with charged fragments $X_{1}$ and $X_{2}$. Such reactions are relevant in astrophysics: the inverse reaction of the photodisintegration of a nucleus is the hadronic capture, which plays an important role in the stellar nucleosynthesis, and is a central part of the proton-proton chain.

This study is carried out with ab initio methods.

The Lorentz integral transform (LIT) method is an ab initio method that can also be used to study inclusive reactions. In fact that it allows to solve a continuum problem with bound state methods. In general, we are interested in the response function $R(E)$ of a given reaction. Instead of directly determining the response functions, its Lorentz integral transform can be considered:

$$
L(\sigma)=\int d E \mathcal{L}(E, \sigma) R(E), \mathcal{L}(E, \sigma)=\frac{1}{\left(E-\sigma_{R}\right)^{2}+\sigma_{I}^{2}}
$$

where the kernel is a Lorentzian with $\sigma=\sigma_{R}+i \sigma_{I}$. This is a transform with a controlled resolution: in fact the parameters $\sigma_{R}$ and $\sigma_{I}$ control the position and width of the Lorentzian, respectively. For inclusive reactions, the transform $L(\sigma)$ can be calculated as the squared norm of a localized state $\tilde{\Psi}$ depending on the operator inducting the reaction. Thanks to these properties, the LIT can be calculated using bound state methods, such as expansions on a complete basis and subsequent diagonalization. Due to the Coulomb barrier the cross section becomes very small close to the threshold. We want to check if the resolution of the LIT calculation can be made sufficiently high in order to allow a stable inversion of the transform at very low energies: near the reaction threshold, the precision of the LIT calculation may be not sufficient because a high density of LIT states is needed, since the width

\footnotetext{
a e-mail: s.deflorian.2@unitn.it
} 


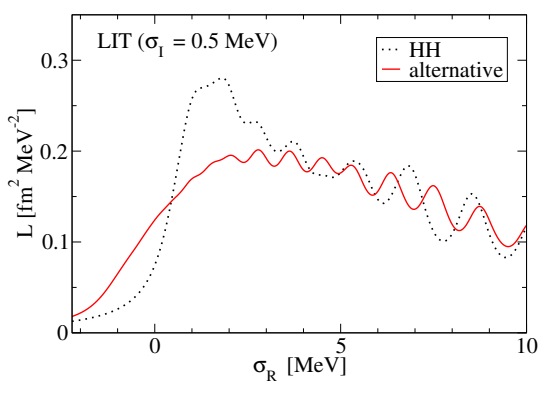

Figure 1. Preliminary results for the LIT of the ${ }^{3} \mathrm{He}$ photodisintegration response function in unretarded dipole approximation, with the Malfliet-Tjon potential. In comparison to the results for the alternative basis (solid line), for the HH basis (dots), with $K_{M A X}=20, N_{M A X}=10$ (as defined in [2]), the strength is shifted to energies above the three-body breakup threshold (here at $\sigma_{R}=0$ ), due to a lack of LIT states in the low-energy region.

$\sigma_{I}$ of each Lorentzian must be sufficiently small to ensure that the low-energy part of the transform doesn't get too strong contributions from other parts of the cross section. Extending the basis leads to a larger number of Hamiltonian eigenstates in general increases the density of such states. It has been observed in recent calculations using a $\mathrm{HH}$ (hyperspherical harmonic) basis for the ${ }^{4} \mathrm{He}$ isoscalar monopole resonance [1] that the density of LIT states is not sufficiently high below the three-body breakup threshold to allow to determine the very small width of the resonance $\left(\Gamma_{\exp }=270 \mathrm{keV}\right)$.

A possible solution to the lack of LIT states near the reaction threshold may be in the use of an alternative basis that is more appropriate to the type of problem studied. Instead of using a $\mathrm{HH}$ basis, we directly use the relative coordinate $\mathbf{r}_{r e l}$ for the fragments. Such a coordinate does not enter explicitly the usual HH formalism. Such a method has been used with success in the description of the above mentioned isoscalar monopole resonance $M(q, \omega)$ of the ${ }^{4} \mathrm{He}$ nucleus [2] (see also the contribution of W. Leidemann to this volume). A similar calculation is presently carried out for the ${ }^{3}$ He photodisintegration (see Fig. 1). After a completion of the LIT calculations, inversions will be performed. One possibility to check the reliability of the inversion of the LIT is to perform a direct calculation of the cross section: such a comparison between the two methods is the aim of the present work. This method in the framework of a $\mathrm{HH}$ approach has been already introduced in [3] and has been further completed using modern realistic potential models (see e.g. [4] and [5]). To perform such a calculation, one has to find the scattering wave function of the product fragments of the reaction. Such a wave function can be written as the sum of an asymptotic and an inner part; the asymptotic part is (almost) known, if the wave functions of each fragment are known, and consists of a linear combination of regular and irregular Coulomb functions:

$$
\left|\Psi_{s c}\right\rangle=\left|\Omega_{a s y m}\right\rangle+\left|\Psi_{i n}\right\rangle,\left|\Omega_{a s y m}\right\rangle=\left[\left|\phi_{A}\right\rangle\left|\phi_{B}\right\rangle\left(Y_{L}^{M}(\theta, \varphi) R_{L}^{R}(r)+T_{L M, L^{\prime} M^{\prime}}^{J} Y_{L}^{M}(\theta, \varphi) R_{L}^{I}(r)\right)\right]_{J, J_{z}} .
$$

Using the Kohn Variational Principle, one finds that the solution satisfies a set of linear equations that have to be solved in order to find the correct wave function which satisfies the Schrödinger equation $(H-E)\left|\Psi_{s c}\right\rangle=0$. Such calculations are currently being performed, and results will be presented in a forthcoming work.

\section{References}

[1] S. Bacca, N. Barnea, W. Leidemann, G. Orlandini, PRL 110, 042503 (2013)

[2] W. Leidemann, PRC 91, 054001 (2015)

[3] B.N. Zakhariev, V.V. Pustovalov, V.D. Efros, Yad. Fiz. 8, 406 (1968)

[4] M. Viviani, A. Kievsky, L.E. Marcucci, S. Rosati, R. Schiavilla, PRC 61, 064001 (2000)

[5] M. Viviani, A. Deltuva, R. Lazauskas, J. Carbonell, A.C. Fonseca, A. Kievsky, L.E. Marcucci, S. Rosati, PRC 84, 054010 (2011) 